\title{
INFLUÊNCIA DO BINÔMIO TEMPO/TEMPERATURA NA ATIVIDADE BACTERICIDA DO EXTRATO AQUOSO DE Moringa oleifera LAM.
}

João Paulo Gomes de Sousa' ${ }^{1}$, Carolini Esmeriz da Rosa ${ }^{2}$, Daiane Evelin dos Santos Assunção ${ }^{3}$, Ed Carlo Paiva Rosa $^{4}$, Jupyracyara Jandyra de Carvalho Barros ${ }^{5}$

\section{RESUMO}

A estabilidade da atividade antimicrobiana do extrato aquoso da semente de Moringa oleifera Lam, em relação ao tempo/temperatura, foi testada a partir da técnica de difusão em disco nas espécies Escherichia coli ATCC 25922, Staphylococcus aureus ATCC 25923 e Pseudomonas aeruginosa ATCC 27853. Os extratos foram colocados em quatro temperaturas diferentes $\left(8^{\circ} \mathrm{C}, 25^{\circ} \mathrm{C}, 37^{\circ} \mathrm{C}\right.$ e $\left.42^{\circ} \mathrm{C}\right)$ e monitorados no tempo zero, três dias, sete dias e dez dias e a única bactéria susceptível a eles foi a Staphylococcus aureus ATCC 25923 (halo de inibição > $13 \mathrm{~mm}$ ). O aumento no volume do extrato não levou ao aumento da efetividade no controle de Staphylococcus aureus ATCC 25923. A partir de três dias, foi observado decréscimo na atividade do extrato, no que se refere à ação bactericida. A temperatura não influenciou o tamanho dos halos de inibição, porém os extratos mantidos a $8{ }^{\circ} \mathrm{C}$ tiveram seu efeito inibidor mantido por maior período de tempo. Os resultados deste estudo indicaram o potencial de aplicação do extrato da semente de Moringa oleifera Lam. como uma alternativa interessante na conservação de alimentos.

Palavras-chave: estabilidade térmica, conservação de alimentos, Staphylococcus aureus

\section{ABSTRACT \\ BINOMIAL INFLUENCE TIME / TEMPERATURE ON THE BACTERICIDA ACTIVITY OF AQUEOUS EXTRACT OF Moringa oleifera LAM.}

The stability of the antimicrobial activity of an aqueous extract of Moringa oleifera Lam. seed versus time / temperature was assayed from the disk diffusion method on the Escherichia coli ATCC 25922, Staphylococcus aureus ATCC 25923 and Pseudomonas aeruginosa ATCC 27853 species. The extracts were placed at four different temperatures $\left(8{ }^{\circ} \mathrm{C}, 25^{\circ} \mathrm{C}, 37^{\circ} \mathrm{C}\right.$ and $\left.42{ }^{\circ} \mathrm{C}\right)$ and monitored at time zero three days, seven days and ten days. The single bacterium was susceptible Staphylococcus aureus ATCC 25923 (inhibition zone $>13 \mathrm{~mm}$ ). The increase extract volume did not lead to increased effectiveness Staphylococcus aureus ATCC 25923 control. From three-day decrease in the extract bactericidal activity was observed. The temperature does not influence the size of the (inhibition zone, though the extracts kept at $8{ }^{\circ} \mathrm{C}$ maintained their inhibitory effect longer. The results of this study indicate the potential application of the seed extract of Moringa oleifera Lam. as an interesting alternative to agriculture, such as in food preservation.

Keywords: thermal stability, food preservation, Staphylococcus aureus.

Recebido para publicação em 06/03/2015. Aprovado em 27/04/2016.

1 - Bacharel em Ciências Biológicas, UFG/Regional Catalão, jotapgomes_26@hotmail.com

2 - Bacharel em Ciências Biológicas, UFG.Regional Catalão, caroliniesmeriz@hotmail.com

3 - Bacharel em Ciências Biológicas, UFG/Regional Catalão, evelin_daiane@hotmail.com

4 - Engenheiro Civil, Professor da UFG/Regional Catalão, edcarlopaiva@yahoo.com.br

5 - Bióloga, Professora da UFG/Regional Catalão, jupyscbarros@ufg.br 


\section{INTRODUÇÃO}

A aplicação de espécies vegetais no controle de patógenos microbianos em detrimento de antimicrobianos convencionais é importante à saúde pública, pois tende a impedir a proliferação de patógenos resistentes (ONUOHA; ALISA, 2013; ONSARE; KAUR; ARORA, 2013).

Dentre os diversos cultivares com propriedade antimicrobiana destaca-se a Moringa oleifera Lam. (JABEEN et al., 2008; NWAIWU; LINGMU, 2011; ONUOHA; ALISA, 2013), capaz de suportar as diferenças edafo-climáticas do Brasil (LO MÔNACO et al., 2010, RAMOS et al., 2010; VIEIRA et al., 2010; PEIXOTO et al., 2011)

Pesquisadores têm investido na prospecção de sementes de Moringa oleifera Lam. como alternativa promissora e de baixo custo para reduzir e/ou eliminar micro-organismos indesejáveis sob o ponto de vista ambiental (NWAIWU; LINGMU, 2011; ALO; ANYIM; ELON, 2012), alimentar (SHARAF et al., 2009; VIEIRA et al., 2010) e/ou clínico (RAHMAN et al., 2010; ABALAKA et al., 2012).

Segundo Gallão et al. (2006), na massa interior das sementes de Moringa oleifera Lam., podem ser detectadas substâncias com perfil antimicrobiano, como glicosídeos moringina e 4-( $\alpha$-L-ramnosilori)-isotiocianato de benzila e 4-( $\alpha$-L-ramnosilori)-fenil-acetonitrila (EILERT; WOLTERS; NAHRSTEDT, 1981).

Nos ensaios de antagonismo in vitro, empregando extratos Moringa oleifera Lam., não há parâmetro mínimo para os halos de inibição (JABEEN et al., 2008; NEPOLEAN; ANITHA; RENITTA, 2009; BUKAR; UBA; OYEYI, 2010; THILZA et al., 2010; SAADABI; ZAID, 2011); a sensibilidade no micro-organismo teste é evidenciada apenas pela presença da zona inibitória. Estudos realizados por Bukar, Uba e Oyeyi (2010) revelaram a ação inibitória de extrato etanólico de sementes de Moringa oleifera Lam. em Escherichia coli, Staphylococcus aureus e Pseudomonas aeruginosa, sendo observados halos de inibição iguais a $8 \mathrm{~mm}, 11 \mathrm{~mm}$ e $6 \mathrm{~mm}$, respectivamente.

Vieira et al. (2010), ao analisarem as propriedades antimicrobianas do extrato aquoso e etanólico de sementes de M. oleifera Lam., consideraram halos de inibição com diâmetro superior a $13 \mathrm{~mm}(\phi>13 \mathrm{~mm})$ representativos da sensibilidade do patógeno ao extrato vegetal em questão. Neste estudo, zonas inibitórias iguais a 27 $\mathrm{mm}$ e $28 \mathrm{~mm}$ foram evidenciados para Escherichia coli e Staphylococcus aureus, nessa ordem.

Saadabi e Zaid (2011) observaram que extratos metanólicos e aquosos de sementes da Moringa oleifera Lam. foram capazes de inibir Escherichia coli, Staphylococcus aureus e Pseudomonas aeruginosa. Para o extrato metanólico, foram obtidos halos de $12 \mathrm{~mm}, 17 \mathrm{~mm}$ e $13 \mathrm{~mm}$, para Escherichia coli, Staphylococcus aureus e Pseudomonas aeruginosa, respectivamente, e para o extrato aquoso os halos foram de $22 \mathrm{~mm}, 48 \mathrm{~mm}$ e $25 \mathrm{~mm}$ na mesma ordem.

A atividade antimicrobiana in vitro de sementes da $M$. oleifera também foi observada quando utilizado extrato aquoso (VIEIRA et al., 2010; SAADABI; ZAID, 2011). Vieira et al. (2010), ao avaliarem $50 \mu \mathrm{L}$ [10 mg da semente (0,05\%)], 100 $\mu \mathrm{L}$ [20 mg da semente $(0,10 \%)], 150 \mu \mathrm{L}$ [30 mg da semente $(0,15 \%)$ e $200 \mu \mathrm{L}$ [10 mg da semente $(0,20 \%)]$ a partir do extrato aquoso de Moringa oleifera Lam. [30 gramas da semente: $150 \mathrm{~mL}$ de água destilada; (1:5)], verificaram que a atividade antimicrobiana foi proporcional ao acréscimo do volume do extrato. Neste estudo, Escherichia coli foi sensível a todos os tratamentos; $0,05 \%$ ( $\phi=16$ $\mathrm{mm}), 0,10 \%$ ( $\phi=17 \mathrm{~mm}), 0,15 \%(\phi=20 \mathrm{~mm}) \mathrm{e}$ $0,20 \%$ ( $\phi=23 \mathrm{~mm})$; o mesmo foi observado para Staphylococcus aureus; o menor halo de inibição foi observado para $0,05 \%(\phi=19 \mathrm{~mm})$.

Os dados reportados acima demonstram a efetividade do extrato obtido da semente de Moringa oleifera Lam. na inibição de microorganismos (ALO; ANYIM; ELOM, 2012), impedindo a formação de resíduos e surgimento de cepas resistentes. Todavia, é necessário certificar a termoestabilidade do caráter antimicrobiano durante o acondicionamento do extrato. Katayon et al. (2004) verificaram que o extrato aquoso de sementes da Moringa oleifera Lam. manteve-se estável à temperatura de $3{ }^{\circ} \mathrm{C}$, ao longo dos cincos dias de armazenamento. Mesmo diante dessa afirmação, ainda são escassas informações sobre a estabilidade dos extratos de moringa, sendo essas informações essenciais para determinar

\section{REVENG}


a viabilidade de sua aplicação. Desse modo, o presente estudo teve como objetivo verificar a estabilidade do extrato aquoso de Moringa oleifera Lam. em relação ao binômio tempo/temperatura.

\section{MATERIAL E MÉTODOS}

Os ensaios in vitro da prospecção do extrato bruto de Moringa oleifera Lam., frente aos micro-organismos Escherichia coli ATCC 25922, Staphylocococcus aureus ATCC 25923 e Pseudomonas aeruginosa ATCC 27853, seguiram as etapas apresentadas na Figura 1.

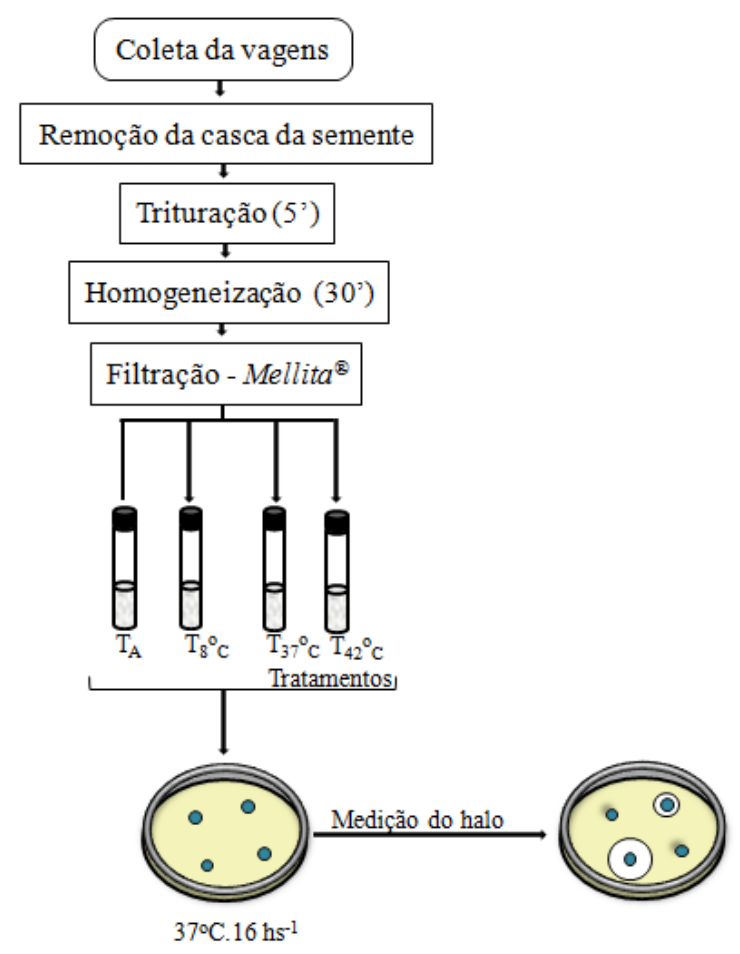

Figura 1. Fluxograma das etapas envolvidas nos ensaios in vitro da atividade antimicrobiana de Moringa oleifera em técnica de disco difusão $\left(\mathrm{T}_{\mathrm{A}}=25\right.$ ${ }^{\circ} \mathrm{C}$ : Extrato Moringa oleifera Lam. mantido em temperatura ambiente; $\mathrm{T}_{8 \mathrm{C}}^{\circ}$ : Extrato Moringa oleifera Lam. submetido ao tratamento térmico de $8{ }^{\circ} \mathrm{C} ; \mathrm{T}_{37^{\circ} \mathrm{C}}$ : Extrato Moringa oleifera Lam. submetido ao tratamento térmico de $37{ }^{\circ} \mathrm{C} ; \mathrm{T}_{42}{ }^{\circ} \mathrm{C}$ : Extrato Moringa oleifera Lam. submetido ao tratamento térmico de $42{ }^{\circ} \mathrm{C}$ ).
A espécie vegetal utilizada nesta pesquisa foi obtida na região do Cerrado goiano. Apenas as vagens devidamente preservadas foram recolhidas e transportadas em sacos plásticos ao Laboratório de Bioquímica e Microbiologia da Universidade Federal de Goiás, Regional Catalão (LABIM/ UFG-CAC).

Sob uma bancada, descontaminada, as vagens foram cuidadosamente abertas $\mathrm{e}$ as sementes retiradas. A parte alada da semente foi removida e a massa branca acondicionada em frascos isentos de sujidades, para posterior trituração em liquidificador de uso doméstico.

Para o preparo do extrato de Moringa oleifera Lam., adotou-se o protocolo proposto por Vieira et al. (2010). Assim, em $150 \mathrm{~mL}$ de água destilada foram adicionadas 30 gramas da semente de Moringa oleifera Lam. (1:5), seguida da homogeneização constante durante 30 minutos em temperatura ambiente $\left(\mathrm{T}_{\mathrm{A}}=25^{\circ} \mathrm{C}\right)$. Em seguida, filtrou-se esse homogenato em papel filtro Melitta $^{\mathbb{B}}$ - $\mathrm{n}^{\circ}$. 103. Posteriormente, esse filtrado foi dividido em tubos de ensaios e mantido em repouso em temperatura ambiente $\left(\mathrm{T}_{\mathrm{A}}=25^{\circ} \mathrm{C}\right)$, e também nas temperaturas de $8{ }^{\circ} \mathrm{C}\left(\mathrm{T}_{8}^{\circ} \mathrm{C}\right), 37{ }^{\circ} \mathrm{C}\left(\mathrm{T}_{37}{ }^{\circ} \mathrm{C}\right)$ e $42{ }^{\circ} \mathrm{C}$ $\left(\mathrm{T}_{42}^{\circ} \mathrm{O}\right)$, durante o período de $0,3,7$ e 10 dias.

Os discos de aproximadamente $6 \mathrm{~mm}$ de diâmetro para o teste de sensibilidade microbiana foram obtidos a partir do filtro Whatman ${ }^{\circledR}-\mathrm{n}^{\circ} .1$ (BUKAR; UBA; OYEYI, 2010), com auxílio de um perfurador de papel, seguida da esterilização em autoclave e secagem a $70{ }^{\circ} \mathrm{C}$. Estes foram impregnados com $50 \mu \mathrm{L}$ [10mg da semente $(0,05 \%)], 100 \mu \mathrm{L}$ [20mg da semente $(0,10 \%)]$, $150 \mu \mathrm{L}$ [30mg da semente $(0,15 \%)]$ e $200 \mu \mathrm{L}$ [40mg da semente $(0,20 \%)$ ] do extrato bruto da Moringa oleifera Lam. (VIEIRA et al., 2010). Para a secagem dos discos, estes foram mantidos em temperatura ambiente $\left(\mathrm{T}_{\mathrm{A}}=25^{\circ} \mathrm{C}\right)$ por um período de 24 horas. Metade dos discos expostos à luz UV e a outra parte isenta desse tratamento.

As culturas overnight de Escherichia coli ATCC 25922, Staphylocococcus aureus ATCC 25923 e Pseudomonas aeruginosa ATCC 27853 foram padronizadas pela escala 0,5 de McFarland $-10^{8}$ células viáveis (BUKAR; UBA; OYEYI, 2010). Após, as culturas foram individualmente semeadas em placas de Petri contendo ágar Müeller Hinton, 
submetidas previamente ao teste de esterilidade. Em condições assépticas e com auxílio de uma pinça, os discos foram depositados na superfície do ágar. A medição do diâmetro do halo foi realizada após 18 a 24 horas de incubação a $37{ }^{\circ} \mathrm{C}$. Foram considerados representativos halos maiores que 13 $\mathrm{mm}$.

Os resultados obtidos foram organizados em tabelas e submetidos à análise estatística descritiva. Índices divulgados em literaturas especializadas, referentes aos testes de sensibilidade das bactérias testadas frente ao extrato de sementes de Moringa oleifera Lam. foram utilizados para comparar os dados obtidos nesta pesquisa.

\section{RESULTADOS E DISCUSSÃO}

Os ensaios com extrato aquoso das sementes de Moringa oleifera Lam. apresentaram resultados diferenciados frente às cepas teste $E$. coli ATCC 25922, S. aureus ATCC 25923 e P. aeruginosa ATCC 27853.

Nas repetições realizadas para todos os tratamentos, o extrato aquoso da semente não inibiu E. coli e P. aeruginosa. Tais informações diferem dos dados obtidos por Saadabi e Zaid (2011), pois esses estudiosos encontraram halo de inibição variando entre 18 e $22 \mathrm{~mm}$ para $E$. coli e 20 e $25 \mathrm{~mm}$ para $P$. aeruginosa, empregando extratos aquosos de $5 \%, 10 \%, 20 \%$ e $40 \%$. Esses estudiosos utilizaram extratos submetidos à prévia liofilização; provavelmente, a remoção da parte líquida do extrato, aliada ao elevado volume do mesmo tenha potencializado a ação das substâncias antimicrobianas.

Vieira et al. (2010) ao utilizarem os mesmos volumes desta pesquisa, $50 \mu \mathrm{L}[10 \mathrm{mg}$ da semente $(0,05 \%)], 100 \mu \mathrm{L}[20 \mathrm{mg}$ da semente $(0,10 \%)], 150$ $\mu \mathrm{L}$ [30mg da semente $(0,15 \%)$ ] e $200 \mu \mathrm{L}$ [40mg da semente $(0,20 \%)$ ] de extrato aquoso de sementes da Moringa oleifera Lam., registraram a efetividade do mesmo para todas os tratamentos em cepas de E. coli isoladas de camarão. Os autores destacaram que a inibição foi proporcional ao acréscimo do volume; $0,05 \%$ ( $\phi=16 \mathrm{~mm}), 0,10 \%$ ( $\phi=17 \mathrm{~mm})$,
$0,15 \%$ ( $\phi=20 \mathrm{~mm})$ e $0,20 \%$ ( $\phi=23 \mathrm{~mm}$ ). Esse dado é interessante, pois, como esses próprios autores ressaltam, E. coli é um importante bioindicador da qualidade sanitária de alimentos e comumente associada a casos de toxinoses alimentares.

Nesta pesquisa, a inibição para $E$. coli não foi registrada em nenhuma repetição, contrapondo os dados de Saadabi e Zaid (2011) e Vieira et al. (2010). Provavelmente, este dado seja devido o uso de estirpes diferentes (CARDOSO et al., 2002) a alteração dos compostos fitoquímicos das plantas resultantes das condições edafo-climáticas (FAROOQ et al., 2007).

S. aureus ATCC 25923 foi o único microorganismo susceptível neste estudo para todos os tratamentos em ao menos um dos períodos de incubação. De modo geral, os maiores halos de inibição foram encontrados no tempo zero, para todas as concentrações/temperatura (Quadro 1). Os discos tratados com UV apresentaram diâmetros de halos de inibição semelhantes àqueles que não foram submetidos a esse tratamento. Vieira et al. (2010), testando o mesmo extrato com a mesma ATCC, obtiveram halos de 19 a $25 \mathrm{~mm}$, corroborando os resultados mostrados no Quadro 1.

As análises realizadas após 3 dias de incubação demonstraram uma redução na efetividade do extrato (Quadro 2). Na terceira repetição desse mesmo tempo, somente o extrato mantido a $8{ }^{\circ} \mathrm{C}$ apresentou zonas de inibição, que variam de $14 \mathrm{~mm}$ a $20 \mathrm{~mm}$. Esse resultado era esperado, pois Katayon et al. (2004) verificou decaimento dos princípios ativos do extrato aquoso de sementes da Moringa oleifera Lam. mantido em temperatura ambiente $\left(\mathrm{T}_{\mathrm{A}}=25^{\circ} \mathrm{C}\right)$; o contrário foi verificado em $3{ }^{\circ} \mathrm{C}$. Jabeen et al. (2008) ressaltam que a efetividade do extrato aquoso da semente é maior a $0{ }^{\circ} \mathrm{C}$.

$\mathrm{O}$ menor halo de inibição foi detectado após sete dias de incubação $(\phi=10 \mathrm{~mm})$, com extrato de $0,05 \%$ mantido a $8{ }^{\circ} \mathrm{C}$ (Quadro 3 ). Nesse tempo, as demais temperaturas não apresentaram efeito inibidor e o maior halo foi observado no disco tratado com UV ( $\phi=19 \mathrm{~mm})$ e apresentando extrato igual $0,15 \%$.

\section{REVENG}


Quadro 1. Atividade bactericida do extrato aquoso de Moringa oleifera Lam., incubado em diferentes temperaturas no tempo zero em $S$. aureus ATCC 25923.

\begin{tabular}{|c|c|c|c|c|c|c|c|c|c|}
\hline \multirow{3}{*}{ REPETIÇÕES } & \multirow{3}{*}{ TEMPERATURA } & \multicolumn{8}{|c|}{ DIÂMETRO DO HALO DE INIBIÇÃO (mm)* } \\
\hline & & \multicolumn{2}{|c|}{$50 \mu \mathrm{L}$} & \multicolumn{2}{|c|}{$100 \mu \mathrm{L}$} & \multicolumn{2}{|c|}{$150 \mu \mathrm{L}$} & \multicolumn{2}{|c|}{$200 \mu \mathrm{L}$} \\
\hline & & $\mathbf{N}_{-U V}$ & $\mathbf{U V}$ & $\mathbf{N}_{- \text {UV }}$ & $\mathbf{U V}$ & $\mathbf{N}_{\text {-UV }}$ & UV & $\mathbf{N}_{-\mathrm{UV}}$ & $\mathbf{U V}$ \\
\hline \multirow{4}{*}{$1^{\mathrm{a}}$} & $8^{\circ} \mathrm{C}$ & 20 & 22 & 21 & 21 & 19 & 20 & 21 & 22 \\
\hline & $25^{\circ} \mathrm{C}$ & 20 & 21 & 22 & 20 & 21 & 22 & 20 & 20 \\
\hline & $37^{\circ} \mathrm{C}$ & 19 & 21 & 21 & 19 & 22 & 21 & 26 & 18 \\
\hline & $42{ }^{\circ} \mathrm{C}$ & 20 & 19 & 21 & 19 & 19 & 22 & 19 & 20 \\
\hline \multirow{4}{*}{$2^{\mathrm{a}}$} & $8{ }^{\circ} \mathrm{C}$ & 19 & 18 & 19 & 18 & 17 & 17 & 18 & 19 \\
\hline & $25^{\circ} \mathrm{C}$ & 18 & 18 & 19 & 19 & 19 & 19 & 20 & 19 \\
\hline & $37^{\circ} \mathrm{C}$ & 18 & 20 & 16 & 19 & 17 & 19 & 17 & 17 \\
\hline & $42{ }^{\circ} \mathrm{C}$ & 20 & 18 & 18 & 16 & 18 & 18 & 18 & 17 \\
\hline \multirow{4}{*}{$3^{a}$} & $8^{\circ} \mathrm{C}$ & 16 & 16 & 20 & 20 & 17 & 18 & 21 & 20 \\
\hline & $25^{\circ} \mathrm{C}$ & 18 & 15 & 18 & 21 & 20 & 20 & 20 & 20 \\
\hline & $37^{\circ} \mathrm{C}$ & 20 & 16 & 20 & 19 & 20 & 20 & 20 & 20 \\
\hline & $42{ }^{\circ} \mathrm{C}$ & 21 & 18 & 19 & 20 & 15 & 22 & 19 & 20 \\
\hline
\end{tabular}

* $50 \mu \mathrm{L}[10 \mathrm{mg}$ da semente $(0,05 \%)], 100 \mu \mathrm{L}$ [20mg da semente $(0,10 \%)], 150 \mu \mathrm{L}[30 \mathrm{mg}$ da semente $(0,15 \%)]$ e $200 \mu \mathrm{L}[40 \mathrm{mg}$ da semente $(0,20 \%)]$ de extrato aquoso de sementes da Moringa oleifera Lam.

Quadro 2. Atividade bactericida do extrato aquoso de Moringa oleifera Lam. em diferentes temperaturas durante três dias de estocagem em S. aureus ATCC 25923.

\begin{tabular}{|c|c|c|c|c|c|c|c|c|c|}
\hline \multirow{3}{*}{ REPETIÇÕES } & \multirow{3}{*}{ TEMPERATURA } & \multicolumn{8}{|c|}{ DIÂMETRO DO HALO DE INIBIÇÃO (mm)* } \\
\hline & & \multicolumn{2}{|c|}{$50 \mu \mathrm{L}$} & \multicolumn{2}{|c|}{$100 \mu \mathrm{L}$} & \multicolumn{2}{|c|}{$150 \mu \mathrm{L}$} & \multicolumn{2}{|c|}{$200 \mu \mathrm{L}$} \\
\hline & & $\mathbf{N}_{\text {-UV }}$ & $\mathbf{U V}$ & $\mathbf{N}_{\text {-UV }}$ & UV & $\mathbf{N}_{\text {-UV }}$ & UV & $\mathbf{N}_{\text {-UV }}$ & UV \\
\hline \multirow{4}{*}{$1^{\mathrm{a}}$} & $8^{\circ} \mathrm{C}$ & 0 & 0 & 0 & 0 & 0 & 0 & 0 & 0 \\
\hline & $25^{\circ} \mathrm{C}$ & 0 & 0 & 0 & 0 & 0 & 0 & 0 & 0 \\
\hline & $37^{\circ} \mathrm{C}$ & 0 & 0 & 0 & 0 & 0 & 0 & 0 & 0 \\
\hline & $42{ }^{\circ} \mathrm{C}$ & 0 & 0 & 0 & 0 & 0 & 0 & 0 & 0 \\
\hline \multirow{4}{*}{$2^{\mathrm{a}}$} & $8^{\circ} \mathrm{C}$ & 16 & 16 & 17 & 17 & 18 & 15 & 16 & 17 \\
\hline & $25{ }^{\circ} \mathrm{C}$ & 17 & 18 & 17 & 19 & 17 & 18 & 15 & 18 \\
\hline & $37{ }^{\circ} \mathrm{C}$ & 14 & 14 & 13 & 14 & 15 & 14 & 14 & 14 \\
\hline & $42{ }^{\circ} \mathrm{C}$ & 16 & 16 & 15 & 18 & 17 & 13 & 19 & 14 \\
\hline \multirow{4}{*}{$3^{a}$} & $8^{\circ} \mathrm{C}$ & 17 & 14 & 20 & 15 & 18 & 15 & 15 & 14 \\
\hline & $25^{\circ} \mathrm{C}$ & 0 & 0 & 0 & 0 & 0 & 0 & 0 & 0 \\
\hline & $37^{\circ} \mathrm{C}$ & 0 & 0 & 0 & 0 & 0 & 0 & 0 & 0 \\
\hline & $42{ }^{\circ} \mathrm{C}$ & 0 & 0 & 0 & 0 & 0 & 0 & 0 & 0 \\
\hline
\end{tabular}

* $50 \mu \mathrm{L}[10 \mathrm{mg}$ da semente $(0,05 \%)], 100 \mu \mathrm{L}[20 \mathrm{mg}$ da semente $(0,10 \%)], 150 \mu \mathrm{L}$ [30mg da semente $(0,15 \%)]$ e $200 \mu \mathrm{L}[40 \mathrm{mg}$ da semente $(0,20 \%)]$ de extrato aquoso de sementes da Moringa oleifera Lam. 
Quadro 3. Atividade bactericida do extrato aquoso de Moringa oleifera Lam. em diferentes temperaturas durante sete dias de estocagem em S. aureus ATCC 25923.

\begin{tabular}{|c|c|c|c|c|c|c|c|c|c|}
\hline \multirow{3}{*}{ REPETIÇÕES } & \multirow{3}{*}{ TEMPERATURA } & \multicolumn{8}{|c|}{ DIÂMETRO DO HALO DE INIBIÇÃO (mm)* } \\
\hline & & \multicolumn{2}{|c|}{$50 \mu \mathrm{L}$} & \multicolumn{2}{|c|}{$100 \mu \mathrm{L}$} & \multicolumn{2}{|c|}{$150 \mu \mathrm{L}$} & \multicolumn{2}{|c|}{$200 \mu \mathrm{L}$} \\
\hline & & $\mathbf{N}_{-\mathrm{UV}}$ & UV & $\mathbf{N}_{-\mathbf{U V}}$ & $\mathbf{U V}$ & $\mathbf{N}_{-\mathrm{UV}}$ & UV & $\mathbf{N}_{-\mathrm{UV}}$ & $\mathbf{U V}$ \\
\hline \multirow{4}{*}{$1^{\mathrm{a}}$} & $8^{\circ} \mathrm{C}$ & 0 & 0 & 0 & 0 & 0 & 0 & 0 & 0 \\
\hline & $25^{\circ} \mathrm{C}$ & 0 & 0 & 0 & 0 & 0 & 0 & 0 & 0 \\
\hline & $37^{\circ} \mathrm{C}$ & 0 & 0 & 0 & 0 & 0 & 0 & 0 & 0 \\
\hline & $42 \mathrm{C}$ & 0 & 0 & 0 & 0 & 0 & 0 & 0 & 0 \\
\hline \multirow{4}{*}{$2^{\mathrm{a}}$} & $8^{\circ} \mathrm{C}$ & 16 & 17 & 18 & 18 & 16 & 19 & 16 & 18 \\
\hline & $25^{\circ} \mathrm{C}$ & 0 & 0 & 0 & 0 & 0 & 0 & 0 & 0 \\
\hline & $37^{\circ} \mathrm{C}$ & 0 & 0 & 0 & 0 & 0 & 0 & 0 & 0 \\
\hline & $42{ }^{\circ} \mathrm{C}$ & 0 & 0 & 0 & 0 & 0 & 0 & 0 & 0 \\
\hline \multirow{4}{*}{$3^{\mathrm{a}}$} & $8^{\circ} \mathrm{C}$ & 10 & 12 & 14 & 13 & 10 & 15 & 12 & 15 \\
\hline & $25^{\circ} \mathrm{C}$ & 0 & 0 & 0 & 0 & 0 & 0 & 0 & 0 \\
\hline & $37^{\circ} \mathrm{C}$ & 0 & 0 & 0 & 0 & 0 & 0 & 0 & 0 \\
\hline & $42{ }^{\circ} \mathrm{C}$ & 0 & 0 & 0 & 0 & 0 & 0 & 0 & 0 \\
\hline
\end{tabular}

* $50 \mu \mathrm{L}[10 \mathrm{mg}$ da semente $(0,05 \%)], 100 \mu \mathrm{L}$ [20mg da semente $(0,10 \%)], 150 \mu \mathrm{L}$ [30mg da semente $(0,15 \%)]$ e $200 \mu \mathrm{L}[40 \mathrm{mg}$ da semente $(0,20 \%)$ ] de extrato aquoso de sementes da Moringa oleifera Lam.

De modo geral, o aumento do volume do extrato não causou aumento de efetividade em $S$. aureus ATCC 25923.

Os resultados obtidos neste trabalho diferiram dos de Vieira et al. (2010), ao empregarem o mesmo tipo de extrato adotado nesta pesquisa. Possivelmente, esses dados estejam associados as características edafo-climáticas das regiões de onde foram retiradas as sementes de moringa.

A instabilidade do extrato é verificada a partir 72 horas de incubação, tal resultado pode ser justificado pelo acúmulo de resíduos orgânicos advindos das reações enzimáticas favorecidas às temperaturas $25{ }^{\circ} \mathrm{C}, 37{ }^{\circ} \mathrm{C}$ e $42{ }^{\circ} \mathrm{C}$. Considerando a estabilidade do extrato incubado a $8{ }^{\circ} \mathrm{C}$, há possibilidade de aplicação do extrato de sementes de Moringa oleifera Lam. em gêneros alimentícios, excessivamente manipulados e comercializados em temperatura de refrigeração.

\section{CONCLUSÕES}

- Os resultados obtidos neste estudo permitiram inferir que o acondicionamento do extrato de sementes de Moringa oleifera Lam. sob baixas temperaturas possibilitou a preservação da sua atividade bactericida durante 7 dias de estocagem, sendo evidente a efetividade do extrato apenas para $S$. aureus ATCC 25923.

\section{REFERÊNCIAS BIBLIOGRÁFICAS}

ABALAKA, M.E.; DANIYAN, S.Y.; ADEYEMO, S.O. The antibacterial evaluation of Moringa oleifera leaf extracts on selected bacterial pathogens. Journal of Microbiology Research, v.2, n.2, p. 1-4, 2012.

ALO, M.N.; ANYIM, C.; ELOM, M. Coagulation and antimicrobial activities of Moringa oleifera seed storage at $3{ }^{\circ} \mathrm{C}$ temperature in turbid water. Pelagia Research Library, v.3, n.2, p.887-894, 2012.

BUKAR, A.; UBA, A.; OYEYI, T.I. Antimicrobial profile of Moringa Oleifera lam. extracts against some food - borne microorganisms, Bayero 
Journal of Pure and Appplied Sciences, v.3, n.1, p.43-48, 2010.

CARDOSO, A.L.S.P.; TESSARI, E.N.S.P.; ZANATTA, G.F. Avaliação da susceptibilidade a antimicrobianos de cepas de Escherichia coli de origem aviária. Arquivos do Instituto Biológico, São Paulo, v.69, n.2, p.1-5, 2002.

EILERT, U.; WOLTERS B.; NAHRSTEDT, P. The antibiotic principle of Moringa Oleifera and Moringa Stenopetala. Planta Medica, v.42, n.1, p.55-61, 1981.

FAROOQ, A. et al. Moringa oleifera: a food plant with multiple medicinal uses. Phytotherapy Research, v.21, n.1, p.17-25, 2007.

GALLÃO, M.I.; DAMASCENO, L.F.; BRITO, E.S. Avaliação química e estrutural da semente de moringa. Revista Ciência Agronômica, Fortaleza, v.37, n.1, p.106-109, 2006.

JABEEN, R.; SHAHID, M.; ASHRAF, M. Microscopic evaluation of the antimicrobial activity of seed extracts of Moringa oleifera. Pakistan Journal of Botany, v.40, n.4, p.13491358, 2008.

KATAYON, S.; NOOR, M.J.M.M.; ASMA, M.; THAMER, A.M.; ABDULLAH, A.G.L.; SULEYMAN, A.M.; AMINUDDIN, M.B.; JHOR, B.C. Effects of storage duration and temperature of Moringa oleifera stock solution on its performance in coagulation. International Journal of Engineering and Technology, v.1, n.2, p.146-151, 2004.

LO MONACO, P.A.V.L.; MATOS, A.T.; RIBEIRO, I.C.A.; NASCIMENTO, F.S.; SARMENTO, A.P. Utilização de extrato de sementes de moringa como agente coagulante no tratamento de água para abastecimento e águas residuárias. Revista Ambiente e Água, v.5, n.3, p.222-231, 2010.

NEPOLEAN, P.; ANITHA, J.; RENITTA, E.R. Isolation, analysis and identification of phytochemicals of antimicrobial activity of
Moringa oleifera Lam. Current Biótica, v.3, n.1, p.33-39, 2009.

NWAIWU N.E.; LINGMU B. Studies on the effect of settling time on coliform reduction using Moringa Oleifera seed powder. Journal of Applied Sciences in Environmental Sanitation, v.6, n.3, p.279-286, sep. 2011.

ONSARE, J.G.; KAUR, H.; ARORA, D.S. Antimicrobial activity of Moringa oleifera from different locations against some human pathogens. Academia Journal of Medicinal Plants, Índia, v.1, n.5, p.80-91, 2013.

ONUOHA, S.C.; ALISA, C.O. Antimicrobial potential of leaf juice and extracts of Moringa oleifera lam against some human pathogenic bacteria. Journal of Pharmacy and Biological sciences, v.5, n.4, p.37-42, 2013.

PEIXOTO, J.R.O.; SILVA, G.C.; COSTA, R.A.; FONTENELLE, J.L.S.; VIEIRA, G.H.F.; FILHO, A.A.F.; VIEIRA, R.H.S.F. In vitro antibacterial effect of aqueous and ethanolic moringa leaf extracts. Asian Pacific Journal of Tropical Medicine, v.4, n.3, p.201-204, 2011.

RAHMAN, M.M. Control of coliform bacteria detected from diarrhea associated patients by extracts of Moringa oleifera. Nepal Medicine College Journal, v.12, n.1, p.12-19, 2010.

RAMOS, L.M.; COSTA, R.S.; MÔRO, F.V.; SILVA, R.C. Morfologia de frutos e sementes e morfofunção de plântulas de Moringa (Moringa oleifera Lam.). Comunicata Scientiae, v.1, n.2, p.156-160, 2010.

SAADABI, A.M.; ZAID, I.E.A. An in vitro antimicrobial activity of Moringa oleifera L. seed extracts against different groups of microorganisms. Australian Journal of Basic and Applied Sciences, v.5, n.5, p.129-134, 2011.

SHARAF, A.M.; EBRAHIUM, M.E.; AMMAR, M.S.; EL-GHANY, M.E.A. Influence of using 
moringa meal flour as meat extender on quality characteristics of beef burger patties during frozen storage. World Journal of Dairy and Food Sciences, v.4, n.1, p.32-40, 2009.

THILZA, I.B.; ISHA, Z.A.; SANI, F.S.; TALLE, M.; JOSEPH, M.B. In vitro antimicrobial activity of water extract of Moringa oleifera leaf stalk on bacteria normally implicated in eye diseases.
Academia Arena, Nigeria, v.2, n.6, p.80-82, 2010.

VIEIRA, G.H.F.; MOURÃO, J.A.; ÂNGELO, A.M.; COSTA, R.A.; VIEIRA, R.H.S.F. Antibacterial effect (in vitro) of Moringa oleifera and Annona muricata against Gram positive and Gram negative bacteria. Revista do Instituto de Medicina Tropical de São Paulo, v.52, n.3, p.129132, 2010. 